\title{
Reflexiones e insumos sobre los Sistemas de Información Gerencial en la gestión de las instituciones de educación superior: Ventajas y desventajas
}

\author{
Thoughts about Information Management Systems at the University of Costa Rica: Advantages and \\ disadvantages of its Administrative System
}

Recibido 20 marzo 2014 • Aceptado 11 noviembre 2014 • Corregido 23 abril 2015

\author{
Walter Araya Garita ${ }^{1}$ \\ Escuela de Lenguas Modernas \\ Universidad de Costa Rica \\ San José, Costa Rica \\ walter.arayagarita@ucr.ac.cr
}

\begin{abstract}
Resumen. El presente trabajo realiza un análisis descriptivo sobre el concepto de Sistemas de Información Gerencial en el contexto de las instituciones de educación superior. Se detallan algunas nociones básicas como son: datos, información, conocimiento y bases de datos, necesarias para poder comprender la necesidad, funcionalidad y éxito de un sistema de información gerencial. Se enfoca en la situación actual de la Universidad de Costa Rica, mediante la descripción de algunas aplicaciones que ha desarrollado la institución, las cuales describen la premisa de la inexistencia de este tipo de sistemas en esta casa de enseñanza. Al mismo tiempo, se clasifican, con base en la información analizada, posibles ventajas y desventajas de los Sistemas de Información Gerencial. Por último, se concluye la importancia de transformar los datos en información, así como de la participación de los diferentes actores que utilizan estos sistemas de acuerdo con las diferentes fuentes bibliográficas consultadas.
\end{abstract}

Palabras clave. Sistemas de Información Gerencial; información; conocimiento; universidad; instituciones de educación superior; tecnología; gestión universitaria

Abstract. This is a descriptive analysis on management information systems at institutions of higher learning. Basic educational management theories are reviewed including, information systems, knowledge and databases required for understanding the need, functionality and success of a management system. This paper also focuses on the current administrative system at the University of Costa Rica and describes applications developed at the institution as an effort to address the lack of such systems. In addition, the advantages and disadvantages of university administrative systems were classified based on the information that was analyzed. It is concluded that information

1 Máster en la Enseñanza del Inglés como Lengua Extranjera de la Universidad de Costa Rica. Estudiante de Maestría Centroamericana en Administración Universitaria, de la Universidad de Costa Rica. Posee Diploma en Administración Educativa de la National University of Educational Planning and Administración, Nueva Delhi, India. Bachiller en Inglés de la Escuela de Lenguas Modernas de la Universidad de Costa Rica. Actualmente labora en la Escuela de Lenguas Modernas de la Universidad de Costa Rica como profesional, investigador y traductor-intérprete del idioma inglés; también ha sido profesor de esta área en esa institución. Además, es miembro de la Comisión de Traductores e Intérpretes Oficiales del Ministerio de Relaciones Exteriores y Culto del Gobierno de Costa Rica. 
management systems are indispensable at institutions for higher learning. The University of Costa Rica cannot ignore this situation and must strategically leverage on the systems it uses in order to convert the data to information in the different academic sectors.

Keywords. Management information systems; information; knowledge; university; institutions of higher education; technology; university management

\section{Introducción}

Las organizaciones ágiles son responsables de tener el correcto conocimiento, en el lugar correcto yen el tiempo correcto. Las instituciones de educación superior, por su naturaleza, están llamadas a crear conocimiento. Son instituciones complejas en su funcionamiento y por ende, en su administración. En el contexto costarricense, las instituciones de educación superior se pueden considerar jóvenes, en comparación con sus contrapartes internacionales. La Decana de estas instituciones es la Universidad de Costa Rica, creada en 1940; actualmente cuenta con un aproximado de cuarenta mil estudiantes y más de diez mil colaboradores entre personal docente y administrativo.

La Universidad de Costa Rica cuenta con una estructura organizativa ya consolidada, a pesar de su relativa juventud. La gerencia de la Universidad de Costa Rica recae sobre la figura del Rector. Además, existe una figura de junta directiva, la cual es nominada comoConsejo Universitario. Dentro de un organigrama complejo, la Rectoría o el Rector cuenta con varias oficinas coadyudantes. Estas oficinas, entre otras, tienen como nombre Centro de Informática y Oficina de Planificación Universitaria. El Centro de Informática se define como "una instancia estratégica, asesora, técnica y de servicio, dedicada a las mejores prácticas para asegurar que la información y tecnología están acordes y soportan los objetivos de la Institución hacia una posición de vanguardia y excelencia" (Centro de Informática, Universidad de Costa Rica, ¿Quiénes somos?, 2014, párr. 1). Como puede observarse, esta instancia universitaria está debidamente normada dentro del contexto de la gestión de la Universidad de Costa Rica, pues su misión y objetivos están delimitados dentro de la estructura organizativa de esta institución de educación superior.

Esta dependencia universitaria es promotora y facilitadora de servicios de tecnología de información y telecomunicaciones (TICS), solo en el ámbito interno de esta institución, con el fin de brindar asesoría y servicio a todas las unidades y entidades que requieran asistencia en las áreas que el Centro de Informática tiene la capacidad para ofrecer.

Por otro lado, existe la Oficina de Planificación Universitaria (OPLAU). Esta oficina tiene como propósito el fortalecimiento y el desarrollo institucional para lograr, de manera eficaz y eficiente, los fines institucionales, con base en un proceso ordenado y sistemático de planificación, congruente con las demandas del entorno (OPLAU, Universidad de Costa Rica, 2014, Objetivos, párr. 1). Esta oficina debe ser la creadora intelectual de los Sistemas de Información Gerencial de la Universidad de Costa Rica. 
A partir de este escenario, se puede comprender que la Universidad de Costa Rica cuenta con la infraestructura administrativa y logística para contar con un sistema de información gerencial; no obstante, en la actualidad no existe, aunque ya se han dado los primeros pasos. A continuación, se discutirán diferentes fuentes que argumentan la necesidad de contar con este tipo de sistemas en cualquier institución, pero se contextualizará en la Universidad de Costa Rica para concluir posibles ventajas y desventajas de la adopción de los Sistemas de Información Gerencial en la institución antes mencionada.

\section{Concepto de sistemas de información}

Definir un sistema de información resulta complejo. Para una organización, de acuerdo con Villarroel, Ochoa y Rincón (2008, p. 2), "un Sistema de Información, constituye uno de los elementos de mayor complejidad con los que puede trabajar el ser humano, dado el gran número de variables con las que puede operar"; por lo que pretender dar una definición exacta que lo englobe en su totalidad, podría no cubrir su significado completo o por el contrario, ser excesivamente global. Sin embargo, como punto de partida se pueden adoptar definiciones lo suficientemente genéricas que sirvan como marco de referencia para su entendimiento, a pesar de su amplitud.

Los Sistemas de Información Gerencial son una necesidad en las instituciones actuales, sin importar su naturaleza. Por ende, las instituciones educativas y en este caso de educación superior, no deben ser la excepción. La actualidad representa una época de la información, por lo que esta debe estar disponible para cualquier usuario. Laudon y Laudon (2013, p. 5) establecen que "un sistema de información es un conjunto de componentes interrelacionados que recolectan (o recuperan), procesan, almacenan y distribuyen información para apoyar la toma de decisiones y el control en una organización". Los sistemas de información son esenciales para las instituciones que trabajan con información, pues se convierten en una herramienta indispensable para una eficaz gestión universitaria, necesaria en este contexto actual, ya que permite el intercambio de ideas, la transferencia del conocimiento y el acceso a la información a terceras partes. También se debe mencionar, el uso de la información para la toma de decisiones.

En la Universidad de Costa Rica, por su tamaño, se genera una gran cantidad y variedad de información (de carácter administrativo, estudiantil, de investigación, acción social, entre otros). Un ejemplo de esta información pueden ser la cantidad de estudiantes matriculados en cursos de idioma portugués y la demanda insatisfecha. Esta información debería estar unida a un sistema de información para la investigación que tome en cuenta este tipo de demanda insatisfecha como indicador para desarrollar un proyecto o por el contrario, ofrecerlo a nivel de extensión docente. Además, se debería unir la información antes mencionada, con una parte de información administrativa, como puede ser la disponibilidad de espacio físico para abrir el proyecto de extensión a la comunidad. La masificación del uso de herramientas tecnológicas 
entre los usuarios de las instituciones de educación superior es una realidad. No es posible visualizar a un estudiante universitario sin acceso a internet, incluso dentro de las aulas.

Para Nava (2007, p. 42), "las TIC han generado una inmensa popularidad en el sentido de simplificarlasactividadesqueserealizan parala difusión sistemáticayautomatizada dela información". Esta tendencia crece cada vez más y los usuarios no esperan menos de las instituciones de educación superior. Es improbable pensar en un estudiante que no demande el uso de aplicaciones de sus universidades para realizar diferentes trámites o acceder a información importante por medio de sus teléfonos inteligentes. Los materiales electrónicos de última generación, auditados y confiables, interconectados con el resto del mundo a través del ciberespacio, son los referentes para estas nuevas generaciones de estudiantes y usuarios de la información. Este fenómeno, lamentablemente, produce una brecha de conocimiento entre generaciones y por ende, renuencia a la implementación de sistemas gerenciales por parte de algunos directivos universitarios.

\section{Información vs. conocimiento}

Los datos por si solos no ayudan en la gestión de las instituciones de educación superior. El uso de estos convertidos en información se transforma en una herramienta en la administración de las universidades. Los autores Laudon y Laudon (2013) manifiestan que las tecnologías de la información son una variable estratégica que las compañías a nivel mundial han empezado a usar de una manera considerable en todos los ámbitos de la empresa. Por ende, en las instituciones de educación superior se deben utilizar a nivel global, en todas sus áreas como lo son la administrativa, la docencia, la acción social y la investigación. Las tecnologías de información agilizan eficientemente los procesos, disminuyendo su dificultad, pero su mayor ventaja es la transmisión de la información que trae implícito el conocimiento, convirtiéndolo en un recurso productivo y estratégico a nivel empresarial. Se considera que la información es uno de los activos más importantes de cualquier compañía; es un valor que se debe saber administrar y del que depende el buen funcionamiento de cualquier empresa.

La información como tal, debe ser clasificada para ser considerada relevante. Ante este enunciado, Gabalán y Vásquez (2012, p. 315), consideran que "cada vez tenemos más información, estamos sometidos a una verdadera sobredosis diaria desde múltiples fuentes". Un ejemplo de estas fuentes es la Internet. Para estos autores los usuarios, en Internet, no navegan; por el contrario, en la Internet naufragan. Por ende, los usuarios de la información caen en una situación negativa. Las personas, en general, consumen mucha más información de la que son capaces de digerir. Sin embargo, al mismo tiempo, continúan exponiendo los autores antes mencionados, la sociedad actual está inconforme con el conocimiento que posee. Ante esta situación, Gabalán y Vásquez (2012) consideran la sencillez para obtener información, la cual es bastante, aunque no necesariamente se transforme en conocimiento. Ellos consideran que es urgente desterrar la creencia que recopilar, distribuir y acumular información equivale a generar conocimiento. 
Otros autores apoyan este enunciado; por ejemplo, para Laudon y Laudon (2013) la información está compuesta por datos que se han moldeado en forma significativa y que representan cierta utilidad para los seres humanos. El concepto de información útil es esencial en la toma de decisiones. En otras palabras, la información no es solo el conjunto de datos o peor aún los datos independientes, sin ser analizados y evaluados. Otros autores refuerzan esta idea de transformar datos en información, como lo son Gabalán y Vasquez (2012), quienes consideran que:

La información supera la capacidad de los datos iniciales al contener significancia y pertinencia; es decir, la información puede definirse como un conjunto de datos significativos y pertinentes que describen sucesos o entidades, entendibles y asimilables por la organización (incluyendo cada uno de sus elementos constitutivos) o el ente que los contiene. (p. 314)

Ante la realidad que podrían enfrentar las organizaciones de contar con gran cantidad de datos sin transformarse en información, es importante cuestionarse cuál es el concepto de información de calidad relevante para la organización. Si las organizaciones no lo hacen, se empieza a acumular datos que, con el paso de tiempo, se hacen obsoletos y difíciles de manejar.

Sin embargo, la información por sí sola no debe ser el fin del uso de las herramientas relacionadas con los sistemas de información, tal y como lo expone Nava (2007), quien reflexiona que existe un segundo nivel y menciona lo siguiente con respecto al conocimiento:

Es más relevante para sostener los negocios que el capital, el trabajo o la tierra. El conocimiento es esencial para la acción, el rendimiento y la adaptación y provee la capacidad para responder a situaciones nuevas que se pueden generar en cualquier contexto educativo. (p. 46)

Desde esta perspectiva, la información solo es útil para cualquier organización si la misma se convierte en uno de los que podría ser el activo más preciado: el conocimiento. Este mismo autor concluye que "desde un punto de vista holístico, el conocimiento forma parte de las ideas, los juicios, los talentos, las relaciones, las perspectivas y los conceptos" (Nava, 2007, p. 46). Desde esta perspectiva, los sistemas de información solo cumplen su objetivo si los datos no solo se transforman en información, sino más bien cuando esta información se convierte en conocimiento.

De igual manera, Gabalán y Vásquez (2012) consideran que la información debe, necesariamente, contar con ciertas propiedades como la relevancia en el contexto específico donde se presente integrar. Además, la información debe ser precisa o exacta en relación con la realidad para que su representación alcance un nivel adecuado de confiabilidad. Este elemento es esencial, pues los directivos universitarios deben ir ganando la confianza de los sistemas de información. La información debe estar completa en lo posible, al menos así se debe tratar para los elementos claves de la decisión. La información debe ser adecuada para que sea considerada tanto en el momento de la entrega por parte del emisor como en su recepción por parte del 
receptor y por último, la información debe ser oportuna o comunicada en el momento de su uso y por ende, debe ser comprensible o entendible para quien la recibe.

Si la información cumple las propiedades antes mencionadas, se puede considerar como conocimiento. Por otro lado, Davenport y Prusak (1998), citados por Nava (2007), explican que la información se transforma en conocimiento mediante los siguientes procesos:

1. La comparación (identificando similitudes y/o diferencias de una situación frente a otras conocidas o similares); 2 . Las consecuencias (identificando las implicaciones que tiene la información en un contexto determinado o en varios, para la toma de decisiones y acciones); 3. La conexión (observando y analizando cómo se relaciona un fragmento de información -o tal vez visualizando su conversión en conocimiento con otros factores-); y 4. La conversación (identificando los significados o las interpretaciones que le dan diferentes personas a una misma información). (p. 136)

Entonces se puede concluir que no es solo recolectar información e ingresarla al sistema, es más que ese proceso. Requiere toda una técnica para clasificar esos datos a los que, erróneamente, se le llama información.

El conocimiento de la información garantizará el éxito de la organización. Al respecto, Valdés (1995, p. 11), citado en Nava (2007, p. 47), indica que "la base del desarrollo de una empresa inteligente está determinada por su capacidad de estructurar y sistematizar su capital intelectual; éste puede sustituir cualquier factor de producción". Por ende, el conocimiento convierte a la institución de educación superior en una organización más eficiente, con mayor capacidad de gestión y con mayores posibilidades de ser exitosa. Nava (2007), por ejemplo, categoriza la gestión del conocimiento en gestión funcional y gestión estratégica:

En la gestión funcional del conocimiento, las organizaciones, consientes de la necesidad de distribuir información, están empleando una serie de técnicas de gestión funcional del conocimiento con la principal preocupación de conectar a las personas con el sistema que se utiliza para la distribución y la transferencia de conocimiento. (p. 47)

La gestión estratégica del conocimiento establece un equilibrio al vincular la creación del conocimiento de una compañía con su estrategia empresarial, prestando atención al impacto de la informática y a la necesidad de diseñar la estructura de la organización en consonancia con esa estrategia.

Además, algunas instituciones muestran preocupación por este elemento de desintegración, por lo que consideran que la información es uno de sus activos más importantes y la ven como un valor (o por lo menos un generador de valor) que se debe saber administrar, pues de la administración de la información depende el buen funcionamiento de la organización. 


\section{Contexto de los sistemas de información en la gestión de la Universidad de Costa Rica}

Para Laudon y Laudon (2013), lo presentando por Nava (2007), solo se daba en la teoría y no en la práctica. Los primeros autores señalan que, en la mayoría de las organizaciones, los archivos de datos y los sistemas marcaban una tendencia a crecer de manera independiente, sin ajustarse a un plan a nivel de toda la empresa. Esta proliferación de sistemas aislados hace pensar que en la universidad se considera una expansión anárquica. Parece ser el caso de la Universidad de Costa Rica, en donde la mayoría de las instancias universitarias no tienen sus sistemas integrados. Por ejemplo, las aplicaciones de la Oficina de Registro e Información Estudiantil no están conectadas con otros sistemas como el de la Oficina de Recursos Humanos. Es importante destacar los esfuerzos realizados hasta el momento en esta universidad, porque aunque sea de manera independiente, se está avanzando, pero se debe cuestionar que son esfuerzos aislados.

Sin embargo, se puede caer en el problema de contar con muchas herramientas que, con el paso del tiempo, se convierten en una carga, puesto que las mismas no son actualizadas y al no existir comunicación entre las diferentes instancias universitarias, se encuentran aplicaciones duplicadas u obsoletas para el contexto actual de la universidad. Es probable que en cinco o diez años, la Universidad de Costa Rica se encuentre con una carga de cientos de programas y aplicaciones muy difíciles de mantener y manejar. Por lo tanto, se generarán diferentes problemas, entre los cuales se puede citar la redundancia e inconsistencia de datos, pues todos son alimentados de maneras diferentes. Además, se va a crear dependencia entre los programas y los datos. Uno de los principales problemas que se puede encontrar, además, es la inflexibilidad, una escasa seguridad de datos y por encima de estos, la incapacidad de compartir datos entre aplicaciones. Puede ser que esta situación se empiece a dar en esta institución de educación superior, debido a que la misma información difiere según donde se consulte, por lo que parece que no es actualizada en tiempo real.

Esta redundancia de datos ocurre cuando diferentes grupos de una organización capturan, de manera independiente, la misma pieza de información y la almacenan por aparte de los demás grupos. Entonces, tanto la Oficina de Planificación de la Universidad de Costa Rica como el Centro de Informática deben tomar posición como entes rectores en el área de las tecnologías de la información, para no crear esfuerzos individuales como hasta el momento se trabaja.

Al realizar un análisis sobre la página web de la universidad, el usuario se encuentra diferentes aplicaciones que, como se ha mencionado anteriormente, parecen no estar conectadas entre sí. A nivel interno, por ejemplo, las aplicaciones para presentar proyectos de investigación, acción social y docencia tienen la misma plataforma; sin embargo, existe un total divorcio para preparar los informes. En cuanto a los usuarios externos, no existen aplicaciones amigables que concentren información sobre planes de estudio y becas. Por el contrario, los visitantes deben hacer nuevas búsquedas para acceder a información que puede estar enlazada 
entre las aplicaciones. De igual manera, el formato de las páginas es heterogéneo y parecen ser páginas de diferentes instituciones, tan solo es necesario visitar dos páginas de dos instancias universitarias como unidades académicas u oficinas administrativas para darse cuenta de ello. Los formatos son diferentes con línea gráfica distinta.

Una de las más visitadas, probablemente, por la demanda que tiene, es la Oficina de Registro e Información Estudiantil, en lo que respecta a sistemas de solicitud de ingreso y matricula a la Universidad. En este sitio web, los usuarios, quienes son estudiantes potenciales tienen la posibilidad de inscribirse en el proceso de admisión para la población mencionada en el primer caso. Para los estudiantes ya admitidos, el sistema les permite matricular los cursos de la oferta académica. Esto se logra cuando la Universidad de Costa Rica enlaza su página web a una base de datos interna. Este caso, por ejemplo, no solo se da en la Universidad de Costa Rica; muchas otras organizaciones utilizan ahora la "web" para poner parte de la información de sus bases de datos internas a disposición de sus clientes y socios de negocios, como son sus estudiantes.

No obstante, en la actualidad, a pesar de contar con toda esa información, el cliente o usuario sigue siendo el dueño de la misma, según la nueva Ley de Protección de la Información. Pero, ¿qué sucede con los otros aspectos mencionados por Laudon y Laudon (2013)? El pago a proveedores, en la Universidad de Costa Rica, no está sistematizado dentro de un sistema de información gerencial. Existe una relación entre los sistemas de la Oficina de Administración Financiera, por medio de sus bases de datos, con la Oficina de Suministros, a través de la aplicación Gestión de Compras (GECO), la cual es utilizada para que los proveedores de la Universidad puedan realizar sus trámites sin la necesidad de presentarse a las instalaciones. Además, es utilizada por las instancias internas para solicitar a la Oficina de Suministros o Proveeduría los bienes necesarios para la buena gestión administrativa y académica. Los usuarios de esta aplicación no son solo funcionarios de la Universidad de Costa Rica, sino también proveedores de materiales e insumos necesarios para la gestión de la academia. Sin embargo, esta aplicación como la anterior, está aislada y con casi nula interacción dentro de un sistema general.

Una de las principales tareas pendientes que tiene la Universidad de Costa Rica es la integración de los sistemas de la Oficina de Recursos Humanos. Es probable que sea un proceso complejo, precisamente por la cantidad de funcionarios y la situación de interinazgo que algunos presentan. Lo más preocupante es que no solo no están integrados a otros sistemas de otras unidades académicas o administrativas, sino también entre ellos mismos. Prueba de ello es una aplicación a la cual debe ingresar el funcionario de la Universidad para realizar su Declaración Jurada de la jornada laboral, la cual debería ver en conjunto con su nombramiento o acción de personal.

Dentro de este análisis, se puede concluir que las aplicaciones antes mencionadas solo funcionan para dar seguimiento a los clientes, en este caso estudiantes. Este aspecto es fundamental pero delicadoal mismo tiempo. Esimportante, pues en la actualidad las instituciones de educación superior están inmersas en una tendencia de procesos de autoevaluación con 
fines de acreditación. Por ende, las diferentes carreras, por medio de las unidades académicas, deben reunir información solicitada por los entes acreditadores. Esta información se obtiene precisamente a partir de la información ingresada por esos "clientes".

\section{Bases de datos}

Para estos procesos deberían ser los mismos usuarios los responsables de suministrar la información para las bases de datos, con el fin de que esta no sea manipulada por terceros y por ende, no se contamina. Las bases de datos son de suma importancia para las organizaciones; al respecto, Laudon y Laudon (2013, p. 15) indican que "las empresas utilizan sus bases de datos para dar seguimiento a las transacciones básicas, como el pago a proveedores, el procesamiento de pedidos, el seguimiento de los clientes y el pago a los empleados". Pero también necesitan las bases de datos para suministrar información que ayudará a la empresa a manejar los negocios de manera más eficiente, y que ayudará a los gerentes y a los empleados a tomar mejores decisiones. Si una empresa desea saber cuál producto es el más popular o quién es su cliente más rentable, puede encontrar la respuesta en los datos.

De igual forma, creen que "todas las empresas, grandes y pequeñas, necesitan una política de información. Los datos de su empresa son un recurso importante, y al usuario no le agradará que los demás hagan con ellos lo que se les antoje" (Laudon y Laudon, 2013, p. 23). El usuario necesitará contar con reglas sobre la manera en que se organizarán y mantendrán los datos, y quién tendrá autorización para verlos o modificarlos.

Una política de información busca especificar las reglas de la organización para compartir, distribuir, adquirir, estandarizar, clasificar e inventariarla información. Una política de información establece procedimientos y responsabilidades determinados, que identifican cuáles usuarios y unidades de la organización pueden compartir información, dónde se puede distribuir esta y quién es responsable de actualizarla y mantenerla.

Es importante destacar que las bases de datos reducen la redundancia y la inconsistencia de estos, al minimizar la cantidad de archivos aislados en los cuales se repite la misma información. Sin embargo, es posible que una base de datos no permita a la organización eliminar por completo la redundancia de ellos, pero puede ayudarle a controlarla.

Las organizaciones enfrentan problemas al no almacenar toda la información en bases de datos. Entre estos problemas se puede mencionar la dependencia de diferentes instancias para recolectar datos, pues en la actualidad, para reunir esa información, hay que enviar correos electrónicos, hacer llamadas, enviar cartas y en algunos casos, esperar hasta días. Además, la información no es flexible; en los datos no se pueden buscar diferentes tendencias, por ejemplo. La seguridad es otra limitante, pues los datos no son custodiados de la mejor manera. 
Por el contrario, las bases de datos permiten trabajar lo que es minería. Según Laudon y Laudon (2013, p. 20), "Ios tipos de información que se pueden obtener a partir de la minería de datos incluyen asociaciones, secuencias, clasificaciones, agrupaciones y pronósticos". De ahí, que esta herramienta, si bien es cierto requiere de una gran inversión de tiempo y recurso humano, se puede convertir en un importante insumo para la gestión universitaria, pues es una fuente primaria, inmediata y confiable de información para la toma de decisiones.

Este procedimiento es bastante meticuloso; una vez que se han capturado y organizado los datos en almacenes de datos y en mercados de datos, los mismos están a disposición para análisis más profundos. Una serie de herramientas permite a los usuarios analizar estos datos para descubrir nuevos patrones, relaciones y conocimientos profundos para orientar la toma de decisiones. "Estas herramientas para consolidar, analizar y dar acceso a extensas cantidades de datos para ayudar a los usuarios a tomar mejores decisiones de negocios con frecuencia se denominan inteligencia de negocios (BI)" (Laudon y Laudon, 2013, p. 17). Por ende, más que los usuarios, las personas gestoras universitarias requieren de estas herramientas para la toma de decisiones, pues de otra manera, las mismas se tomarían sin fundamento o en un contexto muy limitado. En el caso de la Universidad de Costa Rica, al ser una institución grande y compleja, esta información debe ser esencial para el Rector y los diferentes Vicerrectores y Vicerrectoras. En estas circunstancias, las autoridades se ahorran recursos al contar con información instantánea y segura.

Las principales herramientas para la inteligencia de negocios incluyen softwares para consultas e informes de bases de datos, herramientas para análisis de datos multidimensionales (procesamiento analítico en línea) y minería de datos. Desde el punto de vista de varios investigadores, cuando se piensa en la inteligencia como se aplica a los humanos, por lo general, se asocia con la capacidad de la gente para combinar el conocimiento aprendido con nueva información y cambiar comportamientos, con la idea de alcanzar el éxito en su actividad o adaptarse a una nueva situación. De manera similar, la inteligencia de negocios da a las empresas la capacidad de acumular información, desarrollar conocimiento sobre clientes, competidores y operaciones internas, así como cambiar la manera de tomar decisiones para lograr una rentabilidad más alta y otras metas de negocios (Laudon y Laudon, 2013).

\section{Planificación para la implementación de Sistemas de Información}

La planificación juega un papel importante en la implementación de los sistemas de información en las instituciones de educación superior. Los gestores universitarios deben proyectar las necesidades de la institución y de sus usuarios, ya que los sistemas de información en plazos muy cortos se convierten en obsoletos. Así, lo manifiestan diferentes autores como Anniccharico, Diaz y Primera (2003), quienes consideran que:

El inventario de conocimientos generados por la empresa y expresados como tecnología, información y habilidades desarrolladas en el personal, constituyen el Capital Intelectual, el 
cual se traduce como patentes, mejora de procesos, productos, servicios, conocimiento de clientes, proveedores, competencia, entorno y oportunidades. (p. 89)

Además, son la solución de problemas en equipo, comunicación, manejo de conflictos y desarrollo de la inteligencia, pues permiten que los usuarios tengan información de primera mano, procesos más expeditos y una gestión más eficiente. De ahí, la importancia de incorporar este tipo de herramientas a la gestión universitaria, para aplicaciones en procesos de matrícula, compras de activos, sistemas internos de recursos humanos, entre otros.

La planificación figura como la primera función administrativa por ser la base de las demás. Señala Chiavenato (2006), citado por Villarroel et al. (2008, p. 3), que“la planificación es la función administrativa que determina por anticipado cuales son los objetivos que deben alcanzarse y que debe hacerse para conseguirlos". Puede afirmarse entonces, que la planificación constituye un modelo teórico para la acción futura, el cual empieza por la determinación de los objetivos y detalla los planes necesarios para alcanzarlos de la mejor manera posible.

De esta manera, la implementación de sistemas de información, según Anniccharico, Diaz y Primera (2003), no se puede dar de la noche a la mañana. Los pasos para generar un sistema de información son: analizar el sistema de decisión, analizar los requisitos de información, conjuntar las decisiones, diseñar el proceso de información; es decir, requiere de un proceso de planificación específico y coherente con lo que se pretende lograr.

Emery (2004), citado por Villarroel et al. (2008), propone un conjunto de características a tomar en cuenta en el diseño de todo sistema de información:

- Disponibilidad de la información cuando sea necesaria y por los medios adecuados (determinados impresos, papel, pantallas interactivas, acceso remoto, fax, modem, entre otros).

- Suministro de la información de manera"selectiva", evitando sobrecargas e información irrelevante. Supone la sustitución de "cantidad por calidad", es decir información.

- Variedad en la forma de presentación de la información.

- El grado de "Conocimiento" incorporado en el sistema (relaciones preestablecidas entre las informaciones contempladas en el sistema).

- El tiempo de respuesta del sistema: diferencia entre una petición de servicio y su realización.

- Exactitud: conformidad entre los datos suministrados por el sistema y los reales.

- Generalidad: conjunto de funciones disponibles para atender diferentes necesidades.

- Flexibilidad: capacidad de adaptación y/o ampliación del sistema a nuevas necesidades.

- Seguridad: protección contra perdida y/o uso no autorizado del sistema.

- Reserva: nivel de repetición de la información para proteger de perdidas catastróficas de alguna parte del sistema. 
- "Amigabilidad" para con el usuario: grado con que el sistema reduce las necesidades de aprendizaje para su manejo. (p. 2)

Estas características son importantes de considerar, pues no se debe caer en la obsolescencia, monotonía y desconfianza que se encuentran en otras aplicaciones carentes de estas especificaciones.

\section{Ventajas de los sistemas de información en la gestión universitaria}

Son muchas las ventajas que se pueden señalar en cuanto a la implementación de un sistema de información gerencial en las instituciones de educación superior. Primero, se debe señalar que las tecnologías de la información agilizan eficientemente los procesos, disminuyendo su dificultad, pero su mayor ventaja es la transmisión de información que trae implícito el conocimiento, convirtiéndolo en un recurso productivo y estratégico a nivel empresarial. El hacer un trámite o gestión desde la comodidad de su hogar u oficina abarata, en gran medida, los costos, permitiendo a la administración contraer los gastos, pues disminuye la necesidad de recurso humano y facilita al usuario el realizar trámites sin la necesidad de desplazarse. El proceso es más ágil y no se crean filas, las cuales, en la mayoría de los casos, generan una percepción negativa de la organización.

Las organizaciones modernas deben tener como meta invertir adecuadamente sus recursos. Los Sistemas de Información Gerencial son una herramienta que permite lograr este objetivo, pues es transparente, debido a que los datos, en su mayoría, son generados por los mismos usuarios. Además, se ha comprobado que la eficacia de estos permite un menor consumo o gasto de recursos. Su efectividad también permite que sean relevantes, no solo para la organización sino también para los usuarios de externos de la misma.

Otra de las ventajas que se puede mencionar es la solución que estos presentan en cuestión estructural. Por ejemplo, se dejarían de utilizar edificios completos para archivar documentos físicos; en este sentido, las organizaciones hacen uso de espacios electrónicos para archivar su información. Evitan que los usuarios se acerquen a sus instalaciones, pues logran la realización de trámites utilizando diferentes aplicaciones y por ende, se reducen gastos como se mencionó anteriormente.

En el ámbito interno de la organización, los Sistemas de Información Gerencial presentan una oportunidad para difundir los fines, misión y objetivos de la organización. Asimismo, evitan la fragmentación organizativa, pues todo se encuentra centralizado en un solo sistema. Además, la gestión no se aísla, sino es una sola. Une planes de naturaleza estratégica, los cuales permiten que la organización sea más eficiente y no se requieran de jornadas de "socialización" de estos.

Un aspecto importante es la creación de una cultura de autoevaluación que permita que los esfuerzos realizados no se pierdan y no se tenga que empezar desde el inicio cada vez que se 
desarrolle una nueva aplicación, pues como se ha mencionado anteriormente, se deben mantener los datos actualizados y los resultados se deben ver al instante, en el contexto que se desarrolla la gestión. Esta al ser más proactiva, mejora relaciones del ambiente externo, lo que permite superar tensiones y por ende, se logra integrar con otras instituciones nacionales e internacionales.

\section{Desventajas de los sistemas de información en la gestión universitaria}

Son pocas las desventajas que se pueden mencionar para los Sistemas de Información Gerencial dentro de la gestión universitaria. El más relevante podría ser los modelos mentales de los directivos, quienes no se atreven a dar el paso para implementar este tipo de sistemas, lo que se refleja en el retraso que presentan instituciones como la Universidad de Costa Rica cuando se comparan con otras instituciones de educación superior, tanto nacionales e internaciones. Estas personas prefieren mantener a su organización dentro de un esquema más tradicional.

Una segunda desventaja es el factor económico. La implementación de un Sistema de Información Gerencial requiere de un presupuesto robusto, pues el diseño del mismo no solo demanda la parte técnica sino un proceso de planificación, el cual requiere recurso humano calificado y un mantenimiento adecuado, continúo y preventivo, muchas veces inalcanzable para las instituciones de educación superior públicas o poco atractivo, en inversión, para las instituciones privadas.

Algunas veces, una de las desventajas es la inexistencia de una normativa que rija la competencia en materia de información. Al ser una disciplina reciente y al comportarse de una manera cambiante, la normativa no es tan expedita como los cambios del entorno.

Por último, los directivos o gerentes deben estar conscientes que estos procesos demandan un tiempo prudencial. Los mismos no se pueden desarrollar de la noche a la mañana. Por el contrario, es este período el que permite tener un criterio de evaluación del sistema y referencia los cambios que se deben hacer sobre la marcha. La paciencia de los directivos es una debilidad, ya que muchos esperan cambios a corto plazo y el entorno cambia frecuentemente. El problema de muchos directivos universitarios es la falta de información a pesar de que, en muchos casos, hay una sobreoferta de datos que pueden ser transformados en conocimiento, mediante un debido proceso, por medio de los sistemas de información, según sean las necesidades de la organización.

\section{Conclusiones}

A partir del análisis realizado, se concluye que la información por si sola es solo datos, los cuales son pasivos y estáticos. Esta situación no se modifica hasta que las personas le agreguen valor hasta convertirla en conocimiento, el cual es activo y dinámico para la organización, tanto a nivel interno como externo. Por ende, los Sistemas de Información Gerencial deben ser herramientas útiles en ese sentido. Para lograrlo, el sistema debe ser capaz de suministrar información oportuna 
y relevante a las autoridades para la toma de decisiones relativas al funcionamiento de la institución, además de servir de apoyo a la investigación y a la extensión, ya que la complejidad de estos procesos dificulta, en cierta medida, el flujo interno y externo de información.

Los datos solos, por si mismos, no reducen la dosis de ignorancia o el grado de incertidumbre de quien tiene que tomar una decisión. Por ende, en el proceso de planificación se debe cuestionar: ¿Cuánta información necesitan los directivos universitarios? ¿Cuántas personas la piden adecuadamente? ¿Cuántas personas se pueden comunicar con los programadores o personal técnico? La información es un concepto valioso pero no se traduce en inversión directa. El sistema no solo debe ser implementado sino también evaluado. Los criterios deben ser economía o costo, utilidad, oportunidad, corrección, relevancia, excepción, comparatividad y comparabilidad, flexibilidad, comprensión y calidad, confiabilidad, disponibilidad, seguridad. La visión estratégica debe incluir la creación de una conciencia entre todos los participantes del proceso (docentes, administrativos, estudiantes) en cuanto a rol, ventajas y limitaciones. Debe existir claridad de políticas y estrategias para la creación, ejecución y mantenimiento de los sistemas de información; identificación de inquietudes de todos los usuarios; creación, apoyo y financiamiento continuo. Las decisiones debe manejarlas un comité gerencial, el cual debe integrar todos los sistemas socioeducativos existentes.

Los Sistemas de Información Gerencial deben ser utilizados en todos los ámbitos de la gestión de la educación superior. En el ámbito administrativo se deben aprovechar los datos para aplicaciones de recursos humanos, financieros, de planificación, entre otros, tanto a nivel docente como administrativo. Así, por ejemplo, con solo el número de identificación de la persona se debería consignar, no solo información personal sino también académica como proyectos de investigación, docencia y acción social. Otro ejemplo, puede ser la implementación de un registro de uso de recursos como los laboratorios o parqueos, los cuales pueden ser monitoreados en línea.

A nivel estudiantil, los Sistemas de Información Gerencial se presentan como una necesidad prioritaria para la administración universitaria. Datos sobre matrícula, becas, deserción, aprobación de cursos, entre otros, son solo algunos ejemplos del uso que pueden tener estos sistemas.

En un ámbito más académico, las universidades deben utilizar los Sistemas de Información Gerencial en beneficio de la sociedad. El almacenamiento de material informativo y científico, así como la proyección social, debe estar al alcance de la sociedad por medio de aplicaciones que permitan a la población tener acceso sin necesidad de trasladarse a los campus universitarios.

En general, el crecimiento de las instituciones de educación superior y sus necesidades de información requieren la implementación de estos sistemas. La Universidad de Costa Rica es un claro ejemplo, pues se observa una administración "acelerada". Se evidencian procesos 
automatizados, pero individuales, que si bien es cierto benefician a todos los usuarios, no tienen como fin la unidad de la información, la cual con el paso del tiempo se puede convertir en un elemento que atente contra la gestión institucional y no como una herramienta.

\section{Referencias}

Anniccharico, E., Diaz, J. \& Primera, N. (2003). Los sistemas de información en la gerencia universitaria. Revista de Ciencias Sociales (RCS), 9(1), 87-102. Recuperado de: http://www.academia.edu/9512935/Los_ sistemas_de_informaci\%C3\%B3n_en_la_gerencia_universitaria

Gabalán, J. \& Vásquez, F. (2012). La administración de la información como potenciadora de la gestión del proceso investigativo en una institución de educación superior: Una propuesta. Revista Interamericana de Bibliotecología, 35(3), 313-326. Recuperado de: http://www.scielo.org.co/scielo.php?pid=S012009762012000300006\&script=sci_arttext

Laudon, K. \& Laudon, J. (2013). Sistemas de Información Gerencial. Administración de la Empresa Digital. Manuscrito no publicado. Recuperado de: http://www.slideshare.net/jes4791/sistemas-deinformacion-gerencial-12-edicin-kenneth-c-laud-on-jane-p-laudon

Nava, R. (2007). Socialización del conocimiento académico con el uso de tecnologías de información y comunicación (TIC). Revista Venezolana de Información, Tecnología y Conocimiento, 4(3), 41-56. Recuperado de: http://www.scielo.org.ve/scielo.php?script=sci_arttext\&pid $=$ S1690-75152007000300004

Universidad de Costa Rica. Centro de Informática. (2014) ¿Quiénes somos? Recuperado de: http://ci.ucr. ac.cr/quienessomos\#page-title

Universidad de Costa Rica. Oficina de Planificación Universitaria. (2014). Objetivos. Recuperado de: http:// www.oplau.ucr.ac.cr/index.php/quienes-somos/objetivos

Villarroel, J., Ochoa, Y. \& Rincón, H. (s. f.). Sistemas de Información: Herramientas de Planificación en las Universidades Venezolanas. III Congreso Internacional de TIC y Pedagogía-UPEL. Centro de Investigación y Formación IntegralVenezolano. Recuperado de:http://cifiv.blogspot.com/2012/08/ sistemas-de-informacion-herramienta-de.html 